> Depuis les années 1990, les origines génétiques de l'autisme sont mises en avant. Malgré les investissements scientifiques et financiers très importants, moins de $20 \%$ des cas d'autisme ont été attribués à des anomalies génétiques. Ces résultats n'empêchent pourtant pas le discours sur l'origine génétique de l'autisme de se propager laissant dans l'ombre les risques liés à la périnatalité. L'analyse détaillée des résultats et des arguments en faveur d'une forte composante génétique de l'autisme révèle des biais méthodologiques, des biais d'interprétation, des approximations erronées et une couverture médiatique exagérée. Les études réalisées chez les jumeaux illustrent ces biais. Un risque supérieur d'autisme pour les jumeaux monozygotes pourrait être lié à une altération de l'environnement intra-utérin. <

\section{L'autisme : surestimation des origines génétiques}

\section{Brigitte Chamak}

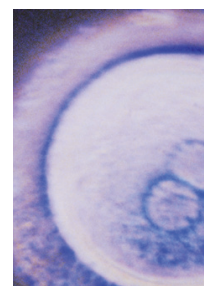

\section{CERMES3-CESAMES}

(Centre de recherche médecine, sciences, santé, santé mentale et société), Inserm U988, દHESS, CNRS UMR8211, Université Paris-Descartes, 45 , rue des Saints-Pères, 75006 Paris Cedex 06, France. brigitte.chamak@parisdescartes.fr

financements très importants ont été consacrés, au niveau international, à la recherche des anomalies chromosomiques et génétiques. Malgré le pourcentage faible de cas reconnus comme relevant d'un problème génétique, ce discours persiste. La médiatisation de l'origine génétique de l'autisme conduit des entreprises peu scrupuleuses à annoncer la commercialisation prochaine d'un test génétique de diagnostic précoce de l'autisme. En 2006, ces pratiques ont fait l'objet

\section{Un syndrome hétérogène aux origines multiples}

L'autisme est défini comme un trouble du développement apparaissant avant l'âge de trois ans, caractérisé par des difficultés de communication et d'interaction sociale associées à des activités stéréotypées et des intérêts restreints. Les manifestations autistiques se caractérisent par une grande hétérogénéité, surtout depuis l'élargissement des critères diagnostiques qui a été introduit dans les classifications des maladies dans les années $1990[1,2]$.

En ce qui concerne l'origine organique de l'autisme, les hypothèses se sont orientées vers des anomalies du développement du système nerveux central et l'implication de facteurs génétiques [3]. Pourtant, lorsqu'une maladie génétique associée à l'autisme est systématiquement recherchée, elle n'est retrouvée que dans moins de $20 \%$ des cas [4]. Les étiologies de l'autisme restent donc, en majorité, inconnues. Néanmoins, depuis les années 1990 , le discours sur la génétique de l'autisme est prédominant et des d'une vive critique formulée par le Comité d'éthique de l'Inserm ${ }^{1}$.

L'implication de facteurs génétiques dans l'autisme est illustrée par des études familiales et par des résultats obtenus à partir de couples de jumeaux où l'un des jumeaux peut être sévèrement atteint et l'autre ne présenter que quelques signes autistiques [5-8]. Les risques liés à la périnatalité restent indéniables [9-11] mais peuvent être limités ou prépondérants, comme dans le cas d'une exposition à l'acide valproïque, la thalidomide, la rubéole ou l'alcool [12]. Ainsi, toutes les atteintes du système nerveux d'origine infectieuse, toxique ou traumatique qui affectent l'enfant pendant la grossesse, l'accouchement ou les premières années de la vie sont susceptibles d'entraîner le développement d'un tableau autistique.

${ }^{1}$ Avis concernant l'annonce de la commercialisation prochaine d'un test génétique de diagnostic précoce de l'autisme. Comité d'éthique de l'Inserm (Ermes). Avis 2006/06 (http://www.ethique.inserm.fr/inserm/ethique.nsf/AllDocumentsByUNID/ ElB2BFA2898BD4BFC125719B005255E5). Voir aussi ces deux articles de Bertrand Jordan dans Médecine/Sciences [28, 29]. 


\section{L'investissement scientifique et financier dans la recherche d'anomalies génétiques}

L'hypothèse génétique de l'autisme jouit depuis plusieurs années d'un large soutien des institutions, des associations de parents d'enfants autistes et des chercheurs. Les investissements financiers ont été très importants. L'International molecular genetics study of autism consortium (IMGSAC) a été fondé en 1994: 300 familles multiplex (familles avec deux sujets atteints ou plus) et 150 familles simplex (un seul sujet autiste et ses deux parents) ont fait l'objet de recherches approfondies. Des gènes candidats sur les chromosomes 2, 7 et 16 ont été plus particulièrement étudiés [13].

Le projet Paris autism research international sibpair study (Paris study) a débuté en 1992. En 2003 ont été identifiés les gènes de la neuroligine 3 et de la neuroligine 4 associés à l'autisme et au syndrome d'Asperger dans deux familles [14]. Cependant, les mutations des neuroligines sont rares et ne sont pas spécifiques de l'autisme puisqu'elles peuvent être associées à des retards mentaux sans autisme [15]. En 2006, des altérations du chromosome $22 q 13$ ont été mises en évidence chez trois familles et des mutations du gène SHANK3 (SH3 and multiple ankyrin repeat domains 3) ont été identifiées [16]. Mais, comme pour les neuroligines, ces résultats ne sont pas spécifiques de l'autisme. Les recherches d'une équipe américaine sur le syndrome de délétion 22q13 (ou syndrome de Phelan McDermid) avaient déjà identifié, en 2003, une délétion du gène SHANK3 et son rôle dans l'apparition de signes neurologiques, mais pas nécessairement autistiques [17].

L'Autism genome project (AGP), lancé en 2002, regroupe plus d'une centaine de chercheurs de 50 centres aux États-Unis, en Europe et au Canada qui ont collecté l'ADN de plus de 1200 familles comportant au moins deux enfants présentant une forme d'autisme. Les anomalies chromosomiques (délétions, duplications) ont été recherchées et la technologie des micropuces d'ADN a été utilisée pour analyser les marqueurs génétiques. Parmi les résultats obtenus, une délétion a été identifiée dans quelques cas sur le chromosome 2 incluant le gène codant pour la neurexine 1 , une protéine d'adhésion qui joue un rôle dans la mise en place des synapses [18]. Récemment, une fréquence plus élevée de CNV (copy number variants) a été trouvée dans environ $6 \%$ des cas d'autisme [30]. Ces variations (non spécifiques de l'autisme) correspondent à des duplications ou délétions de segments de gènes de petite taille.

D'autres centres et institutions, dont AGRE (Autism genetic resource exchange) et le Center for human genetics autism research (Duke university medical center) sont orientés vers l'identification de facteurs génétiques impliqués dans l'autisme. Le Centre de recherche en génétique humaine de l'Université Vanderbilt (Tennessee, États-Unis) a travaillé à partir de 500 familles et a identifié 96 single nucleotide polymorphism (SNP). La région du chromosome 5 pl4.1 a été ciblée. Ce locus se situe entre deux gènes ( $C D H 9$ et $\mathrm{CDH10}$ ) qui codent pour les cadhérines neuronales, protéines d'adhésion qui jouent un rôle dans les connexions entre neurones [19].

Tous ces efforts scientifiques et financiers ont permis d'identifier de nouvelles anomalies mais toujours dans des cas rares, ce qui ne permet aucunement de conclure à une forte héritabilité de l'autisme.

\section{La forte composante héréditaire de l'autisme remise en question}

Faible pourcentage des syndromes génétiques avec manifestations autistiques

Plusieurs maladies génétiques rares sont associées à des manifestations autistiques. Parmi ces syndromes figurent celui de I'X fragile (gène FMR1 : fragile $X$ mental retardation 1 ), la sclérose tubéreuse de Bourneville (gènes TSC1 : tuberous sclerosis 1 ou TSC2), le syndrome de Rett (mutation du gène MCP2 : methyl CpG binding protein 2), les syndromes d'Angelman et de Prader-Willi (délétion 15q11-q13, gène UBE3A : ubiquitin protein ligase E3A), de Smith-Lemli-Opitz (anomalie du métabolisme du cholestérol, gène DHCR7: 7-dehydrocholesterol [7-DHC] reductase), de Smith-Magenis (microdélétion 17q11.2); on trouve aussi la phénylcétonurie non traitée, la neurofibromatose, les syndromes de Sotos, de Cowden et de Bannayan-Riley-Ruvalcab, de Cornelia de Lange, les maladies de San Filippo. D'autres pathologies génétiques rares peuvent être associées à l'autisme: le syndrome de Turner, la trisomie 21, les syndromes de Williams, de Joubert, de Moebius, de Cohen ( $\mathrm{COH}$ gene), de Lujan-Fryns, d'Aarskog, l'hypomélanose d'Ito, la dystrophie myotonique de Steiner, certaines maladies mitochondriales, le syndrome de Charge, le syndrome de Phelan McDermid (microdélétion 22q13) et le déficit en adénylo-succinate lyase (anomalie de la synthèse des purines) $[3,4,20]$. Il est donc important, lors de la démarche diagnostique de l'autisme, de rechercher ces maladies qui requièrent les compétences de cliniciens qui en connaissent les signes cliniques, ce qui en facilite la détection, surtout lorsqu'une maladie métabolique est suspectée et qu'un régime peut permettre de pallier les anomalies.

Cependant, tous ces syndromes ne correspondent qu'à un faible pourcentage des cas d'autisme (moins de $20 \%$ ). L'exemple de l'étude Paris study est significatif de ce point de vue: entre 2003 et 2005, 202 patients atteints d'autisme ou de troubles apparentés [4] appartenant à 176 familles ont été vus en consultation génétique et étudiés en cytogénétique. La recherche de microdélétions et duplications n'a révélé que 8 anomalies (4 de la région 15q11-q13, 2 délétions 22q11 [syndrome de DiGeorge], une délétion 22q13 et une duplication $7 q 11$ [syndrome de Williams] et 7 mutations géniques [4].

\section{Un facteur de risque supérieur chez les jumeaux monozygotes} L'analyse détaillée des résultats et des arguments en faveur d'une forte composante génétique de l'autisme révèle une exagération de la 
couverture médiatique mais aussi des biais méthodologiques, des biais d'interprétation et des approximations qui se sont révélés erronés. Les études réalisées avec des jumeaux illustrent ces biais.

Les études Paris Study, AGP et IMGSAC [13, 14, 18, 30] et les revues de la littérature [3, 21, 22] rapportent que les recherches menées auprès des jumeaux monozygotes révèlent une concordance ${ }^{2}$ de 60 à $92 \%$. La première publication citée [5] ne concerne que 21 paires de jumeaux. Dans cet article, Folstein et Rutter [5] critiquaient les études antérieures qui selon eux présentaient des biais importants puisque le nombre de jumeaux monozygotes était bien supérieur à celui des jumeaux dizygotes, alors que, dans la population générale, ces derniers sont deux fois plus nombreux. Ces auteurs décidèrent de comparer 11 paires de jumeaux monozygotes et 10 paires de jumeaux dizygotes parmi lesquels 14 enfants présentaient un autisme typique et 11 un tableau clinique d'autisme atypique. Un retard mental était identifié chez la moitié des enfants diagnostiqués et un tiers avaient un QI normal aux tests non verbaux. Sur les 10 paires de jumeaux dizygotes, aucune n'était concordante pour l'autisme alors que 4 des 11 paires de jumeaux monozygotes l'étaient. C'est à partir de ces 4 cas que le pourcentage de $36 \%$ de concordance a été calculé. Les auteurs ont ensuite recherché tout ce qui pouvait évoquer des signes d'anomalies cognitives chez les enfants et ont considéré que 9 des 11 paires de jumeaux monozygotes (82\%) présentaient des anomalies cognitives, impliquant souvent le langage, et un seul sur les 10 jumeaux dizygotes. Ce sont sur ces petits effectifs et sur des particularités cognitives que le chiffre de $82 \%$ de concordance s'est propagé.

L'importance des facteurs génétiques dans l'étiologie de l'autisme avait été mise en avant par Folstein et Rutter [5] qui insistaient pourtant sur la fréquence supérieure des anomalies de langage (et non de l'autisme proprement dit) chez les jumeaux monozygotes par rapport aux hétérozygotes. Le risque supérieur d'autisme pour les jumeaux monozygotes, indépendamment des facteurs génétiques, n'a pas été pris en compte par Folstein et Rutter en 1977 [5], ni par Ritvo et al. en 1985 [6], Steffenbourg et al. en 1989 [7], ou Bayley et al. en 1995 [8], malgré les résultats des études antérieures qui indiquaient un nombre plus élevé de jumeaux monozygotes dans les familles étudiées (22 versus 10) [5].

${ }^{2}$ Si le taux de concordance de l'autisme est de 60 à $92 \%$, cela veut dire que chez les monozygotes, quand il y en a un qui est atteint, 60 à $92 \%$ des frères ou sœurs, issus du même œuf, présentent des caractéristiques autistiques (ils ne sont pas forcément autistes).
Une étude publiée en 2001 portant sur des familles avec deux enfants autistes (cohorte $A G R \varepsilon$ ) a mis en évidence une fréquence beaucoup plus élevée de jumeaux que dans la population générale, 4 à 5 fois plus de jumeaux dizygotes et 10 fois plus de jumeaux monozygotes [23]. La gémellité représenterait donc un facteur de risque pour l'autisme, risque d'autant plus grand pour les jumeaux monozygotes, c'est-à-dire issus du même œuf, qui peuvent se trouver en compétition pour les nutriments et l'espace. L'environnement intra-utérin n'est pas identique que les jumeaux soient mono- ou dizygotes [24, 25].

La cohorte Paris study confirme la fréquence supérieure de jumeaux dans les familles avec deux enfants autistes: sur les 79 paires d'enfants présentant des caractéristiques autistiques (définition élargie), 11 étaient des jumeaux, dont 9 monozygotes. Cette fréquence de jumeaux monozygotes est 14 fois plus élevée dans cette cohorte que dans la population générale [26].

Les différentes études sur l'autisme réalisées avec des jumeaux [58] ne tiennent pas compte de ce facteur de risque supérieur chez les jumeaux monozygotes. Du fait des nombreux biais d'interprétation et des incertitudes concernant l'environnement intra-utérin, la gémellité ne peut servir de base objective pour des études qui concluent, à tort, aux origines génétiques prédominantes $[25,27]$. Les cas d'autisme syndromique ont démontré l'implication d'anomalies génétiques dans un nombre de cas limités. Les études épidémiologiques réalisées en Suède [9], au Danemark [10] et en Australie [11] mettent en évidence une association entre les facteurs de risque périnataux et l'augmentation du nombre de cas d'autisme. Les étiologies de l'autisme sont donc à rechercher, pour chaque cas, dans les signes cliniques évocateurs de syndromes génétiques connus, les anomalies de la croissance intra-utérine, l'exposition à des toxiques pendant la grossesse et les événements indésirables durant l'accouchement [27]. Évoquer les facteurs environnementaux pour moduler le discours en génétique ne suffit pas et ne permet pas d'identifier les éléments précis à même de perturber le développement d'un embryon. Ce n'est qu'au cas par cas que les chercheurs et les cliniciens pourront trouver des réponses satisfaisantes. Dans la mesure où l'hétérogénéité des causes d'autisme a été démontrée, les stratégies de recherche en génétique qui consistent à faire abstraction de cette hétérogénéité en analysant les anomalies communes dans le génome de milliers de familles ont trouvé leurs limites. $\diamond$

\section{CONFLIT D’INTÉRÊTS}

L'auteur déclare n'avoir aucun conflit d'intérêts concernant les données publiées dans cet article.

\section{SUMMARY}

Autism: overestimation of the genetic origins

Since the 1990s the genetic causes of autism have been brought to the fore. Despite scientific efforts and huge fundings, less than $20 \%$ of cases of autism have been linked to genetic abnormalities. These results do not slow the spread of discourse on the genetic origin of autism, and the concomitant neglect of risks linked to perinatal factors. A detailed analysis of results and assertions in favour of a strong 
genetic origin of autism reveals methodological biases, misinterpretations and erroneous approximations, as well as an exaggerated media coverage. Studies on twins are illustrative of these biases. The recent demonstration of an excess of twins among sibling pairs with autism, and especially a 10 -fold increase for monozygotic twins compared with the general population frequencies, has questioned the relevance of conclusions from earlier twin studies. Indeed, if being a twin is a risk factor for autism, then there may be an upwards bias in estimates of the genetic contribution to autism, and the intrauterine environment, including competition for nutrients, has been neglected. $\diamond$

\section{RÉFÉRENCES}

1. Chamak $B$, Cohen $D$. L'autisme : vers une nécessaire révolution culturelle. Med Sci (Paris) 2003; 19: 1152-9.

2. Silverman C. Critical review. Fieldwork on another planet: social science perspectives on the autism spectrum. Biosocieties $2008 ; 3: 325-41$.

3. Jamain S, Betancour C, Giros B, et al. La génétique de l'autisme. Med Sci (Paris) $2003 ; 19: 1081-90$

4. Paris Study: http://www.gs-im3.fr/autism/newsletter2006.pdf

5. Folstein S, Rutter M. Infantile autism: a genetic study of 21 twin pairs. J Child Psychol Psychiatry 1977 ; 18 : 297-321.

6. Ritvo ER, Freeman BJ, Mason-Brothers A, et al. Concordance for the syndrome of autism in 40 pairs of afflicted twins. Am J Psychiatry 1985 ; $142: 74-7$.

7. Steffenburg S, Gillberg C, Hellgren L, et al. Twin study of autism in Denmark, Finland, Iceland, Norway and Sweden. J Child Psychol Psychiatry $1989 ; 30: 405-16$.

8. Bailey A, Le Couteur A, Gottesman I, et al. Autism as a strongly genetic disorder: evidence from a British twin study. Psychol Med 1995; $25: 63-77$.

9. Hultman C, Sparén P, Cnattingius S. Perinatal risk factors for infantile autism. Epidemiology $2002 ; 13: 417-23$.

10. Larsson HJ, Eaton WW, Madsen KM, et al. Risk factors for autism: perinatal factors, parental psychiatric history, and socioeconomic status. Am J Epidemiol 2005 ; 161 : 916-25.
11. Leonard H, Nassar N, Bourke J, et al. Relation between intrauterine growth and subsequent intellectual disability in a ten-year population cohort of children in western Australia. Am J Epidemiol 2008 ; 167 : 103-11.

12. Landrigan PJ. What causes autism? Exploring the environmental contribution. Curr Opin Pediatr $2010 ; 22: 219-25$.

13. IMGSAC. A genomewide screen for autism: strong evidence for linkage to chromosomes $2 q, 7 q$, and 16p. Am J Hum Genet $2001 ; 69: 570-81$.

14. Jamain $\mathrm{S}$, Quach $\mathrm{H}$, Betancour $\mathrm{C}$, et al. Mutations of the $X$-linked genes encoding neuroligins NLGN3 and NLGN4 are associated with autism. Nat Genet $2003 ; 34: 27-9$.

15. Laumonnier F, Bonnet-Brilhault F, Gomot M, et al. X-linked mental retardation and autism are associated with a mutation in the NLGN4 gene, a member of the neuroligin family. Am J Hum Genet $2004 ; 74: 552-7$.

16. Durand CM, Betancur C, Boeckers TM, et al. Mutations in the gene encoding the synaptic scaffolding protein SHANK3 are associated with autism spectrum disorders. Nat Genet 2007 ; $39: 25-7$.

17. Wilson HL, Wong ACC, Shaw SR, et al. Molecular characterisation of the 22q13 deletion syndrome supports the role of haploinsufficiency of SHANK3/PROSAP2 in the major neurological symptoms. J Med Genet 2003; $40: 575-84$.

18. Szatmari P, Paterson AD, Zwaigenbaum L, et al. Mapping autism risk loci using genetic linkage and chromosomal rearrangements. Nat Genet 2007 ; 39 : 319-28.

19. Wang $\mathrm{K}$, Zhang $\mathrm{H}, \mathrm{Ma} \mathrm{D}$, et al. Common genetic variants on $5 \mathrm{p} 14.1$ associate with autism spectrum disorders. Nature $2009 ; 459: 528-33$.

20. Cohen D, Pichard N, Tordjman S, et al. Specific genetic disorders and autism: clinical contribution towards their identification. J Autism Dev Disord 2005 ; 35 : 103-16.

21. Folstein SE, Rosen-Sheidley B. Genetics of autism: complex aetiology for a heterogeneous disorder. Nat Rev Genet $2001 ; 2: 943-55$.

22. Xu J, Zwaigenbaum L, Szamari P, et al. Molecular cytogenetics of autism. Curr Genomics 2004 ; $5: 347-64$.

23. Greenberg DA, Hodge SE, Sowinski J, et al. Excess of twins among affected sibling pairs with autism: implications for the etiology of autism. Am J Hum Genet 2001 ; 69 : 1062-7.

24. Lenoir P, Malvy J, Bodier-Rethore C. L'autisme et les troubles du développement psychologique. Paris: Masson, $2003: 240$ p.

25. Phillips DIW. Twin studies in medical research: can they tell us whether diseases are genetically determined? Lancet $1993 ; 341: 1008-9$.

26. Betancur $C$, Leboyer $M$, Gillberg $C$. Increased rate of twins among affected sibling pairs with autism. Am J Hum Genet $2002 ; 70$ : 1381-3.

27. Newschaffer C, Fallin D, Lee NL. Heritable and nonheritable risk factors for autism spectrum disorders. Epidemiol Rev $2002 ; 24: 137-53$.

28. Jordan B. Demandez le test de l'autisme! Med Sci (Paris) $2005 ; 21: 886-7$.

29. Jordan B. L'autisme d'une entreprise. Med Sci (Paris) 2007, $23: 215-6$.

30. Pinto D, Pagnamenta AT, Klei L, et al. Functional impact of global rare copy number variation in autism spectrum disorders. Nature 2010, 9 juin (online).

\section{TIRÉS À PART}

B. Chamak 